\title{
Erratum
}

\section{Equality of the Bulk and Edge Hall Conductances in a Mobility Gap}

\author{
A. Elgart $^{1}$, G.M. Graf ${ }^{2}$, J.H. Schenker ${ }^{2}$ \\ 1 Department of Mathematics, Stanford University, Stanford, CA 94305-2125, USA \\ 2 Theoretische Physik, ETH Zürich, 8093 Zürich, Switzerland
}

Received: 9 August 2005 / Accepted: 11 August 2005

Erratum published online: 5 November 2005 - (c) Springer-Verlag 2005

Commun. Math. Phys. 259, 185-221 (2005)

In Sect. 3.6, "Proof of Lemma 4," the min-max principle is applied incorrectly to estimate the distribution function of $M_{\zeta}$ on the way to proving (3.37), i.e., that

$$
\sum_{\zeta \in \tilde{\mathcal{E}}_{\Delta}} M_{\zeta}<\infty
$$

However. there is a simple proof of (3.37) based on the following estimate obtained in the penultimate line of the second equation following (3.37):

$$
M_{\zeta}^{-2} \geq \frac{1}{4 D_{\varepsilon}^{2}}\left(\psi_{\zeta}, e^{(\mu-2 \varepsilon)|x|} \psi_{\zeta}\right)
$$

Indeed, we then have

$$
M_{\zeta} \leq 2 D_{\varepsilon}\left[\left(\psi_{\zeta}, e^{\delta|x|} \psi_{\zeta}\right)\right]^{-\frac{1}{2}} \leq 2 D_{\varepsilon}\left(\psi_{\zeta}, e^{-\frac{1}{2} \delta|x|} \psi_{\zeta}\right)
$$

with $\delta=\mu-2 \varepsilon$. In the last step we have applied Jensen's inequality with the convex function $t \rightarrow t^{-\frac{1}{2}}$. As $\left\{\psi_{\zeta}: \zeta \in \tilde{\mathcal{E}}_{\Delta}\right\}$ are ortho-normal, we conclude that

$$
\sum_{\zeta \in \tilde{\mathcal{E}}_{\Delta}} M_{\zeta} \leq 2 D_{\varepsilon} \operatorname{tr} e^{-\frac{1}{2} \delta|x|}<\infty,
$$

which is (3.37). 